\title{
Evasion of No-Hair Theorems and Novel Black-Hole Solutions in Gauss-Bonnet Theories
}

\author{
G. Antoniou, A. Bakopoulos, and P. Kanti \\ Department of Physics, University of Ioannina, Ioannina GR-45110, Greece
}

(Received 12 September 2017; revised manuscript received 13 December 2017; published 30 March 2018)

\begin{abstract}
We consider a general Einstein-scalar-Gauss-Bonnet theory with a coupling function $f(\phi)$. We demonstrate that black-hole solutions appear as a generic feature of this theory since a regular horizon and an asymptotically flat solution may be easily constructed under mild assumptions for $f(\phi)$. We show that the existing no-hair theorems are easily evaded, and a large number of regular black-hole solutions with scalar hair are then presented for a plethora of coupling functions $f(\phi)$.
\end{abstract}

DOI: 10.1103/PhysRevLett.120.131102

Introduction.-The existence or not of black holes associated with a nontrivial scalar field in the exterior region has attracted the attention of researchers over a period of many decades. Early on, a no-hair theorem [1] appeared that excluded static black holes with a scalar field, but this was soon outdated by the discovery of black holes with Yang-Mills [2] or Skyrme fields [3]. The emergence of additional solutions where the scalar field had a conformal coupling to gravity [4] led to the formulation of a novel nohair theorem [5] (for a review, see [6]). Recently, this argument was extended to the case of standard scalar-tensor theories [7], and a new form was proposed that covers the case of Galileon fields [8].

However, both novel forms of the no-hair theorem $[5,8]$ were shown to be evaded: the former in the context of the Einstein-dilaton-Gauss-Bonnet theory [9] and the latter in a special case of shift-symmetric Galileon theories [10-12]. A common feature of the above theories was the presence of the quadratic Gauss-Bonnet (GB) term defined as $R_{\mathrm{GB}}^{2}=R_{\mu \nu \rho \sigma} R^{\mu \nu \rho \sigma}-4 R_{\mu \nu} R^{\mu \nu}+R^{2}$, in terms of the Riemann tensor $R_{\mu \nu \rho \sigma}$, the Ricci tensor $R_{\mu \nu}$, and the Ricci scalar $R$. In both cases, basic requirements of the no-hair theorems were invalidated, and this paved the way for the construction of the counterexamples.

Here, we consider a general class of scalar-GB theories, of which the cases $[9,11]$ constitute particular examples. We demonstrate that black-hole solutions, with a regular horizon and an asymptotically flat limit, may in fact be constructed for a large class of such theories under mild only constraints on the coupling function $f(\phi)$ between the scalar field and the GB term. We address the requirements of both the old and novel no-hair theorems, and we show

Published by the American Physical Society under the terms of the Creative Commons Attribution 4.0 International license. Further distribution of this work must maintain attribution to the author(s) and the published article's title, journal citation, and DOI. Funded by SCOAP ${ }^{3}$. that they are not applicable for this specific class of theories. In accordance with the above, we then present a large number of exact, regular black-hole solutions with scalar hair for a variety of forms for the coupling function.

The Einstein-scalar-GB theory.-We first consider the following action functional

$$
S=\frac{1}{16 \pi} \int d^{4} x \sqrt{-g}\left[R-\frac{1}{2} \partial_{\mu} \phi \partial^{\mu} \phi+f(\phi) R_{\mathrm{GB}}^{2}\right],
$$

which describes a generalized gravitational theory containing the Ricci scalar curvature $R$, a scalar field $\phi$, and the quadratic Gauss-Bonnet term $R_{\mathrm{GB}}^{2}$. The latter, being a total derivative in four dimensions, is coupled to $\phi$ through a coupling function $f(\phi)$. The form of the latter quantity may be inspired from either string theory [13] or Horndeski theory [14]. Note that, in this work, we employ units in which $G=c=1$.

By varying the action (1) with respect to the metric tensor $g_{\mu \nu}$ and the scalar field $\phi$, we derive the gravitational field equations and the equation for the scalar field, respectively. These have the covariant form

$$
\begin{aligned}
G_{\mu \nu} & =T_{\mu \nu}, \\
\nabla^{2} \phi+\dot{f}(\phi) R_{\mathrm{GB}}^{2} & =0,
\end{aligned}
$$

where $G_{\mu \nu}$ is the Einstein tensor, and a dot denotes the derivative with respect to the scalar field. Also,

$$
\begin{aligned}
T_{\mu \nu}= & -\frac{1}{4} g_{\mu \nu} \partial_{\rho} \phi \partial^{\rho} \phi+\frac{1}{2} \partial_{\mu} \phi \partial_{\nu} \phi \\
& -\frac{1}{2}\left(g_{\rho \mu} g_{\lambda \nu}+g_{\lambda \mu} g_{\rho \nu}\right) \eta^{\kappa \lambda \alpha \beta} \tilde{R}_{\alpha \beta}^{\rho \gamma} \nabla_{\gamma} \partial_{\kappa} f,
\end{aligned}
$$

where $\tilde{R}_{\alpha \beta}^{\rho \gamma}=\eta^{\rho \gamma \sigma \tau} R_{\sigma \tau \alpha \beta}=\epsilon^{\rho \gamma \sigma \tau} R_{\sigma \tau \alpha \beta} / \sqrt{-g}$. Note that the energy-momentum tensor $T_{\mu \nu}$ receives contributions from both the scalar field and the Gauss-Bonnet term. 
In the context of the above theory, we will seek spherically symmetric solutions, with a line element

$d s^{2}=-e^{A(r)} d t^{2}+e^{B(r)} d r^{2}+r^{2}\left(d \theta^{2}+\sin ^{2} \theta d \varphi^{2}\right)$,

which describes regular, static, asymptotically flat black holes. Our analysis will also investigate the general constraints that the coupling function $f(\phi)$ needs to obey in order for these solutions to arise.

By employing the line element (5), Einstein's equations take the explicit form

$$
\begin{aligned}
4 e^{B}\left(e^{B}+r B^{\prime}-1\right)= & \phi^{\prime 2}\left[r^{2} e^{B}+16 \ddot{f}\left(e^{B}-1\right)\right] \\
& -8 \dot{f}\left[B^{\prime} \phi^{\prime}\left(e^{B}-3\right)-2 \phi^{\prime \prime}\left(e^{B}-1\right)\right],
\end{aligned}
$$

$$
\begin{aligned}
4 e^{B}\left(e^{B}-r A^{\prime}-1\right)=-\phi^{\prime 2} r^{2} e^{B}+8\left(e^{B}-3\right) \dot{f} A^{\prime} \phi^{\prime}, & \\
e^{B}[ & \left.r A^{\prime 2}-2 B^{\prime}+A^{\prime}\left(2-r B^{\prime}\right)+2 r A^{\prime \prime}\right] \\
= & -\phi^{\prime 2} r e^{B}+8 \phi^{\prime 2} \ddot{f} A^{\prime}+4 \dot{f}\left[\phi^{\prime}\left(A^{\prime 2}+2 A^{\prime \prime}\right)\right. \\
& \left.+A^{\prime}\left(2 \phi^{\prime \prime}-3 B^{\prime} \phi^{\prime}\right)\right],
\end{aligned}
$$

while the scalar equation reads

$$
\begin{aligned}
& 2 r \phi^{\prime \prime}+\left(4+r A^{\prime}-r B^{\prime}\right) \phi^{\prime} \\
& \quad+\frac{4 \dot{f} e^{-B}}{r}\left[\left(e^{B}-3\right) A^{\prime} B^{\prime}-\left(e^{B}-1\right)\left(2 A^{\prime \prime}+A^{\prime 2}\right)\right]=0 .
\end{aligned}
$$

In the above, the prime denotes differentiation with respect to $r$; throughout this work, we assume that the scalar field shares the symmetries of the spacetime.

Equation (7) may take the form of a second-order polynomial with respect to $e^{B}$, which can then be solved to give $e^{B}=\left(-\beta \pm \sqrt{\beta^{2}-4 \gamma}\right) / 2$, where

$$
\beta=\frac{r^{2} \phi^{\prime 2}}{4}-\left(2 \dot{f} \phi^{\prime}+r\right) A^{\prime}-1, \quad \gamma=6 \dot{f} \phi^{\prime} A^{\prime} .
$$

Then, eliminating $B$ from the set of the remaining equations (6), (8), and (9), we may form a system of two independent ordinary differential equations of second order for the functions $A$ and $\phi$,

$$
A^{\prime \prime}=\frac{P}{S}, \quad \phi^{\prime \prime}=\frac{Q}{S},
$$

where the functions $P, Q$, and $S$ are lengthy expressions of $\left(r, \phi^{\prime}, A^{\prime}, \dot{f}, \ddot{f}\right)$.

We will now demonstrate that our set of equations, with a general coupling function $f(\phi)$, allows for the construction of a black-hole solution with a regular horizon, provided that $f$ satisfies certain constraints. For a spherically symmetric spacetime, the presence of a horizon is realized for $e^{A} \rightarrow 0$, as $r \rightarrow r_{h}$, or equivalently for $A^{\prime} \rightarrow \infty$ : the latter will be used in our analysis as an assumption, but it will be shown to follow from the former. On the other hand, the regularity of the horizon amounts to demanding that $\phi$, $\phi^{\prime}$, and $\phi^{\prime \prime}$ remain finite in the limit $r \rightarrow r_{h}$. Then, assuming that $A^{\prime} \rightarrow \infty$ while $\phi^{\prime}$ remains finite [15], Eqs. (11) take the approximate forms

$A^{\prime \prime}=-\frac{r^{4}+4 r^{3} \phi^{\prime} \dot{f}+4 r^{2} \phi^{2} \dot{f}^{2}-24 \dot{f}^{2}}{r^{4}+2 r^{3} \phi^{\prime} \dot{f}-48 \dot{f}^{2}} A^{\prime 2}+\cdots$,

$\phi^{\prime \prime}=-\frac{\left(2 \phi^{\prime} \dot{f}+r\right)\left(r^{3} \phi^{\prime}+12 \dot{f}+2 r^{2} \phi^{2} \dot{f}\right)}{r^{4}+2 r^{3} \phi^{\prime} \dot{f}-48 \dot{f}^{2}} A^{\prime}+\cdots$.

Focusing on the second of the above equations, we observe that $\phi^{\prime \prime}$ diverges at the horizon if $f(\phi)$ is either zero or left unconstrained. However, $\phi^{\prime \prime}$ may be rendered finite if either one of the two expressions in the numerator of Eq. (13) is zero close to the horizon.

If we assume that $\left(2 \phi^{\prime} \dot{f}+r\right)=0$ close to the horizon, then a careful inspection of our equations reveals that, in that case, $\phi^{\prime \prime} \simeq \sqrt{A^{\prime}} / \dot{f}$. Thus, for $\phi^{\prime \prime}$ to remain finite, we must demand that $\dot{f} \rightarrow \pm \infty$, near the horizon. This may be easily shown to lead to either a divergent or a trivial scalar field near the horizon, for every elementary form of $f(\phi)$ we have tried. Therefore, for the construction of a regular horizon in the presence of a nontrivial scalar field, we are led to consider the second choice: $r^{3} \phi^{\prime}+12 \dot{f}+2 r^{2} \phi^{2} \dot{f}=0$. This may be easily solved to yield

$$
\phi_{h}^{\prime}=\frac{r_{h}}{4 \dot{f}_{h}}\left(-1 \pm \sqrt{1-\frac{96 \dot{f}_{h}^{2}}{r_{h}^{4}}}\right),
$$

where all quantities have been evaluated at $r_{h}$. To ensure that $\phi_{h}^{\prime}$ is real, we must impose the following constraint on the coupling function

$$
\dot{f}_{h}^{2}<\frac{r_{h}^{4}}{96} .
$$

Turning now to Eq. (12), and using the constraint (14), we find that the coefficient of $A^{\prime 2}$ simplifies to -1 . Then, upon integration with respect to $r$, we obtain $A^{\prime}=$ $\left(r-r_{h}\right)^{-1}+\mathcal{O}(1)$, which, in accordance with our initial assumption, diverges close to the horizon. Integrating once more and putting everything together, we may write the near-horizon solution as

$$
\begin{gathered}
e^{A}=a_{1}\left(r-r_{h}\right)+\cdots, \\
e^{-B}=b_{1}\left(r-r_{h}\right)+\cdots, \\
\phi=\phi_{h}+\phi_{h}^{\prime}\left(r-r_{h}\right)+\phi^{\prime \prime}\left(r-r_{h}\right)^{2}+\cdots .
\end{gathered}
$$


The above describes a regular black-hole horizon in the presence of a scalar field provided that $\phi^{\prime}$ and the coupling function $f$ satisfy the constraints (14) and (15).

We will now show that a general coupling function $f(\phi)$ for the scalar field does not interfere with the requirement for the existence of an asymptotically flat limit for the spacetime (5). We will assume the following power-law expressions for the metric functions and scalar field, in the limit $r \rightarrow \infty$,

$e^{(A, B)}=1+\sum_{n=1}^{\infty} \frac{\left(p_{n}, q_{n}\right)}{r}, \quad \phi=\phi_{\infty}+\sum_{n=1}^{\infty} \frac{d_{n}}{r}$.

Upon substitution in the field equations, we may determine the arbitrary coefficients $\left(p_{n}, q_{n}, d_{n}\right)$. In fact, $p_{1}$ and $d_{1}$ remain arbitrary, and we associate them with the ArnowittDeser-Misner mass and scalar charge, respectively: $p_{1} \equiv$ $-2 M$ and $d_{1}=D$. Then, the asymptotic solutions for the metric functions and scalar field read

$$
\begin{aligned}
e^{A}= & 1-\frac{2 M}{r}+\frac{M D^{2}}{12 r^{3}}+\frac{24 M D \dot{f}+M^{2} D^{2}}{6 r^{4}}+\cdots, \\
e^{B}= & 1+\frac{2 M}{r}+\frac{16 M^{2}-D^{2}}{4 r^{2}}+\frac{32 M^{3}-5 M D^{2}}{4 r^{3}} \\
& +\frac{16 M\left(48 M^{3}-13 M D^{2}-24 D \dot{f}\right)+3 D^{4}}{48 r^{4}}+\cdots, \\
\phi= & \phi_{\infty}+\frac{D}{r}+\frac{M D}{r^{2}}+\frac{32 M^{2} D-D^{3}}{24 r^{3}} \\
& +\frac{12 M^{3} D-24 M^{2} \dot{f}-M D^{3}}{6 r^{4}}+\cdots .
\end{aligned}
$$

It is in order $\mathcal{O}\left(1 / r^{4}\right)$ that the explicit form of the coupling function $f(\phi)$ first makes its appearance-indeed, the higher-curvature GB term is expected to have a minor contribution at large distances where the curvature is small. It is in the near-horizon regime that the GB term mainly works to support a nontrivial scalar field and thus a charge $D$, which then modifies the metric compared to the Schwarzschild case.

Let us now turn our attention to the no-hair theorems that forbid the existence of black-hole solutions in the presence of a scalar field, i.e., the existence of solutions that smoothly connect the near-horizon and far-away asymptotic solutions found above. We will start with the "novel" no-hair theorem developed by Bekenstein [5]. Assuming positivity and conservation of energy, he demonstrated that the asymptotic forms of the $T_{r}^{r}$ component of the energymomentum tensor near the horizon and at infinity could never be smoothly matched. That argument proved beyond doubt that there were no black-hole solutions in the context of a large class of minimally coupled-to-gravity scalar field theories. Here, we will show that the coupling of the scalar field to the quadratic GB term causes the complete evasion of Bekenstein's theorem. This may be in fact realized for a large class of scalar field theories, with the previously studied exponential [9] and linear [11] GB couplings comprising special cases of our present argument.

The energy-momentum tensor $T_{\mu \nu}$ satisfies the equation of conservation $D_{\mu} T_{\nu}^{\mu}=0$ due to the invariance of the action (1) under coordinate transformations. Its $r$ component may take the explicit form

$$
\left(T_{r}^{r}\right)^{\prime}=\frac{A^{\prime}}{2}\left(T_{t}^{t}-T_{r}^{r}\right)+\frac{2}{r}\left(T_{\theta}^{\theta}-T_{r}^{r}\right),
$$

where the relation $T_{\theta}^{\theta}=T_{\varphi}^{\varphi}$ has been used due to the spherical symmetry. The nontrivial components of the energy-momentum tensor $T_{\mu \nu}$ for our theory (1) with a generic coupling function $f$ are

$$
\begin{gathered}
T_{t}^{t}=-\frac{e^{-2 B}}{4 r^{2}}\left\{\phi^{\prime 2}\left[r^{2} e^{B}+16 \ddot{f}\left(e^{B}-1\right)\right]\right. \\
\left.-8 \dot{f}\left[B^{\prime} \phi^{\prime}\left(e^{B}-3\right)-2 \phi^{\prime \prime}\left(e^{B}-1\right)\right]\right\}, \\
T_{r}^{r}=\frac{e^{-B} \phi^{\prime}}{4}\left[\phi^{\prime}-\frac{\left.8 e^{-B}\left(e^{B}-3\right) \dot{f} A^{\prime}\right],}{r^{2}}\right] \\
T_{\theta}^{\theta}=-\frac{e^{-2 B}}{4 r}\left\{\phi^{\prime 2}\left(r e^{B}-8 \ddot{f} A^{\prime}\right)\right. \\
\left.-4 \dot{f}\left[\phi^{\prime}\left(A^{\prime 2}+2 A^{\prime \prime}\right)+A^{\prime}\left(2 \phi^{\prime \prime}-3 B^{\prime} \phi^{\prime}\right)\right]\right\} .
\end{gathered}
$$

We will first investigate the profile of $T_{r}^{r}$ at infinity: using the asymptotic expansions (19), we easily find that $-T_{t}^{t} \simeq-T_{\theta}^{\theta} \simeq T_{r}^{r} \simeq \phi^{2} / 4+\mathcal{O}\left(1 / r^{6}\right)$. Since the metric function $e^{A}$ there adopts a constant value $\left(e^{A} \rightarrow 1\right)$, the dominant contribution to the right-hand side of Eq. (21) is

$$
\left(T_{r}^{r}\right)^{\prime} \simeq \frac{2}{r}\left(T_{\theta}^{\theta}-T_{r}^{r}\right) \simeq-\frac{1}{r} \phi^{2}+\cdots .
$$

Therefore, at asymptotic infinity, the $T_{r}^{r}$ component is positive and decreasing, in agreement with [5], since the GB term is insignificant in this regime.

In the near-horizon regime, $r \rightarrow r_{h}$, the $T_{r}^{r}$ component (23) takes the approximate form

$$
T_{r}^{r}=-\frac{2 e^{-B}}{r^{2}} A^{\prime} \phi^{\prime} \dot{f}+\mathcal{O}\left(r-r_{h}\right) .
$$

The above combination is finite but not negative definite as in [5]. In fact, the $T_{r}^{r}$ component is positive definite, since close to the black-hole horizon, $A^{\prime}>0$, and $\dot{f} \phi^{\prime}<0$ according to Eq. (14). Therefore, the existence of a regular black-hole horizon in the context of the theory (1) automatically evades one of the two requirements of the novel no-hair theorem.

Turning finally to the expression for $\left(T_{r}^{r}\right)^{\prime}$ and employing the energy-momentum components (22)-(24) in Eq. (21), we obtain the following expression, in the limit $r \rightarrow r_{h}$, 


$$
\begin{aligned}
\left(T_{r}^{r}\right)^{\prime}= & e^{-B} A^{\prime}\left[-\frac{r \phi^{\prime 2}}{4 Z}-\frac{2\left(\ddot{f} \phi^{\prime 2}+\dot{f} \phi^{\prime \prime}\right)}{r Z}\right. \\
& \left.+\frac{4 \dot{f} \phi^{\prime}}{r^{2}}\left(\frac{1}{r}-e^{-B} B^{\prime}\right)\right]+\mathcal{O}\left(r-r_{h}\right),
\end{aligned}
$$

where we have defined $Z \equiv r+2 \dot{f} \phi^{\prime}$. Close to the blackhole horizon, Eq. (14) guarantees that $\dot{f} \phi^{\prime}<0$, while $Z>0$. Employing also the metric functions behavior $A^{\prime}>0$ and $B^{\prime}<0$, we conclude that $\left(T_{r}^{r}\right)^{\prime}$ is negative in the near-horizon regime if a sole additional constraint, namely, $\ddot{f} \phi^{\prime 2}+\dot{f} \phi^{\prime \prime}>0$, is satisfied. This may be alternatively written as $\left.\partial_{r}\left(\dot{f} \phi^{\prime}\right)\right|_{r_{h}}>0$ and merely demands that the negative value of the quantity $\left.\left(\dot{f} \phi^{\prime}\right)\right|_{r_{h}}$, necessary for the existence of a regular black-hole solution, should be constrained away from the horizon. This is in fact the only way for the matching of the two asymptotic solutions (16)-(18) and (19) to be realized. Therefore, for $\left.\partial_{r}\left(\dot{f} \phi^{\prime}\right)\right|_{r_{h}}>0$, the $T_{r}^{r}$ component is positive and decreasing also near the horizon regime. As a result, both requirements of the novel no-hair theorem [5] do not apply in this theory, and thus it can be evaded.

The older version of the no-hair theorem for scalar fields [1], which employs the scalar equation of motion, also fails to exclude the existence of black-hole solutions in our theory (1): multiplying the scalar equation (3) by $f(\phi)$ and integrating over the black-hole exterior region, we obtain the integral constraint

$$
\int d^{4} x \sqrt{-g} f(\phi)\left[\nabla^{2} \phi+\dot{f}(\phi) R_{\mathrm{GB}}^{2}\right]=0 .
$$

Integrating by parts the first term, the above becomes

$$
\int d^{4} x \sqrt{-g} \dot{f}(\phi)\left[\partial_{\mu} \phi \partial^{\mu} \phi-f(\phi) R_{\mathrm{GB}}^{2}\right]=0 .
$$

The boundary term $\left[\sqrt{-g} f(\phi) \partial^{\mu} \phi\right]_{r_{h}}^{\infty}$ vanishes both at the horizon (due to the $e^{(A-B) / 2}$ factor) and at infinity (due to the $\partial^{\mu} \phi$ factor). Since $\phi=\phi(r)$, the first term in Eq. (29) gives $\partial_{\mu} \phi \partial^{\mu} \phi=g^{r r}\left(\partial_{r} \phi\right)^{2}>0$ throughout the exterior region. Also, for the metric (5), the GB term has the explicit form

$R_{\mathrm{GB}}^{2}=\frac{2 e^{-2 B}}{r^{2}}\left[\left(e^{B}-3\right) A^{\prime} B^{\prime}-\left(e^{B}-1\right)\left(2 A^{\prime \prime}+A^{\prime 2}\right)\right]$.

Employing the asymptotic solutions near the horizon (16) and (17) and at infinity (19), we may easily see that the GB term takes on a positive value at both regimes. Therefore, in the simplest possible case where both $f(\phi)$ and $R_{\mathrm{GB}}^{2}$ are sign definite, Eq. (29) allows for black-hole solutions with scalar hair for every choice of the coupling function that merely satisfies $f(\phi)>0$.

In order to demonstrate the validity of the aforementioned arguments, we have numerically solved the system of equations (11) and produced a large number of blackhole solutions with scalar hair. The solutions for the scalar field are depicted in Fig. 1 for a variety of forms of the coupling function $f(\phi)$ : exponential, odd and even power law, and odd and even inverse power law. These forms are all simple, natural choices to keep the GB term in the fourdimensional theory. For easy comparison, the coupling constant in all cases has been set to $\alpha=0.01$ and the nearhorizon value of the field to $\phi_{h}=1$. For $f(\phi)=\left(\alpha e^{\phi}\right.$, $\left.\alpha \phi^{2}, \alpha \phi^{3}\right)$, which all have $f_{h}^{\prime}>0$, our constraint (14) leads to a negative $\phi_{h}^{\prime}$; for $f(\phi)=\left(\alpha e^{-\phi}, \alpha \phi^{-1}, \alpha \phi^{-4}\right)$, which have $f_{h}^{\prime}<0$, Eq. (14) demands a positive $\phi_{h}^{\prime}$; the decreasing and increasing, respectively, profiles are clearly depicted in Fig. 1. In all cases, for a given value of $\phi_{h}$, Eq. (14) uniquely determines the quantity $\phi_{h}^{\prime}$. The integration of the system (11) with initial conditions $\left(\phi_{h}, \phi_{h}^{\prime}\right)$ then leads to the presented solutions. The positivity and decreasing profile of the $T_{r}^{r}$ component, necessary features for the evasion of the novel no-hair theorem, are clearly seen in Fig. 2. It is worth mentioning that the second constraint, $\left.\partial_{r}\left(\dot{f} \phi^{\prime}\right)\right|_{r_{h}}>0$, is automatically satisfied for every solution found and does not need any further action or fine tuning of the free parameters. We finally note that, for $\phi>0$, all the above forms of $f(\phi)$ satisfy also the constraint $f(\phi)>0$, derived above for the evasion of the old no-hair theorem.

For a given coupling function $f(\phi)$ and fixed $\left(\alpha, \phi_{h}\right)$, the constraint (15) dictates that there is a lower bound for the horizon radius of the derived black-hole solutions given by $r_{h}^{2}>4 \sqrt{6}\left|\dot{f}_{h}\right|$ and thus a lower bound on their mass. This characteristic has been noted in the exponential-coupling case $[9,16]$ and comprises a generic feature of all the GB black holes found here, which distinguishes them from their General Relativity (GR) analogues. Thus, in the smallmass limit, observable effects may include deviations from standard GR in the calculation of the bending angle of light, the precession observed in near-horizon orbits, and the spectrum from their accretion disks [17]. The emission of scalar radiation strongly depends on the existing coupling

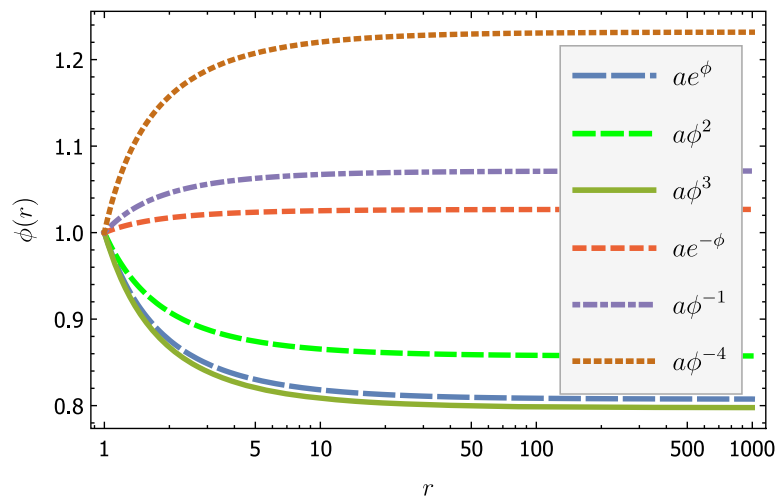

FIG. 1. The scalar field $\phi$ for different coupling functions $f(\phi)$, for $a=0.01$ and $\phi_{h}=1$. 


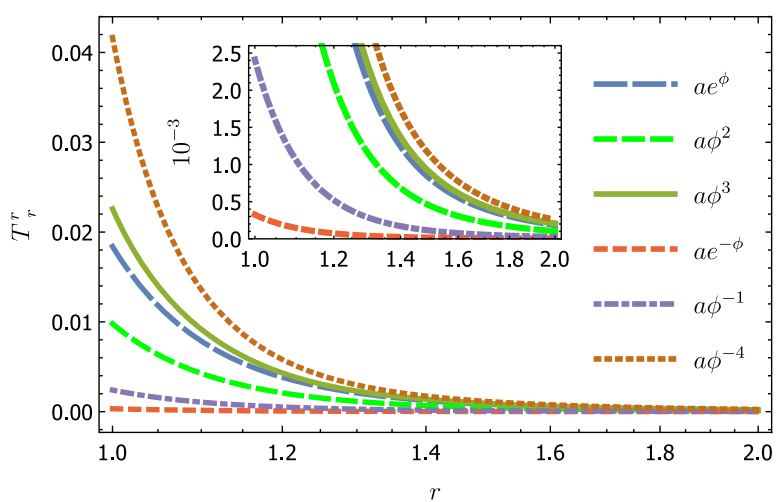

FIG. 2. The $T_{r}^{r}$ component for different coupling functions $f(\phi)$, for $a=0.01$ and $\phi_{h}=1$.

of the scalar field to ordinary matter, while the measurement of the characteristic frequencies of the quasinormal modes (especially the polar sector) will also help to distinguish these solutions from their GR analogues [18]. Finally, the detection of gravitational waves from black-hole or neutron-star mergers may also help to impose bounds on the parameters of the theory, provided that the scalar charge is significant and their physical distance is small.

The scalar-GB theory.-Finally, we investigate whether a regular black-hole solution can arise as the result of the synergy between only the scalar field $\phi$ and the GB term. To this end, we ignore all terms in the field equations coming from the Ricci scalar and attempt to construct a near-horizon, regular solution similar to that of Eqs. (16)(18). In the absence of all $R$-related terms in the field equations, the components of $T_{\mu \nu}$ should vanish. If we assume again that, as $r \rightarrow r_{h}, \phi^{\prime}$ remains finite while $A^{\prime}$ diverges, Eq. (23) yields $e^{B} \simeq 3+\mathcal{O}\left(1 / A^{\prime}\right)$; this clearly does not describe a black hole. Alternatively, demanding that $e^{B} \rightarrow \infty$, as $r \rightarrow r_{h}$, Eq. (23) may be solved for $A^{\prime}$ to give $A^{\prime} \simeq r^{2} \phi^{\prime} / 8 \dot{f}+\mathcal{O}\left(e^{-B}\right)$. Using this, Eqs. (22) and (24) form a system of two differential equations for $B$ and $\phi$. In the limit $r \rightarrow r_{h}$, we find the behavior

$$
\begin{aligned}
B^{\prime} & =-\frac{2}{r} e^{B}+\mathcal{O}\left(e^{-B}\right), \\
\phi^{\prime \prime} & =-\frac{e^{B}}{r} \phi^{\prime}+\mathcal{O}\left(e^{-B}\right) .
\end{aligned}
$$

Equation (31) leads to the solution $e^{-B}=2 \ln \left(r / r_{h}\right)$, which does point toward the existence of a horizon. However, for this horizon to be regular, Eq. (32) demands that $\phi^{\prime}\left(r_{h}\right)=0$. But then, $\phi^{\prime \prime}\left(r_{h}\right)$ is also zero, leading to a constant scalar field outside the horizon. In this case, the GB term does not contribute to the field equations and the above solution disappears.

Conclusions. - In the context of a general Einsteinscalar-GB theory with an arbitrary coupling function $f(\phi)$, we have demonstrated that the emergence of regular black-hole solutions is a generic feature: the explicit form of $f(\phi)$ affects very little the asymptotically flat limit at infinity, while a regular horizon is formed provided that $\phi_{h}^{\prime}$ and $f(\phi)$ satisfy the constraints (14) and (15).

The existing no-hair theorems were shown to be evaded under mild assumptions on $f(\phi)$. The old no-hair theorem [1] is easily evaded for $f(\phi)>0$, while the novel no-hair theorem [5] is nonapplicable if the same constraint (14) holds. Based on this, we have produced a large number of regular black-hole solutions with nontrivial scalar hair for arbitrary forms of the coupling function $f(\phi)$. They are all characterized by a minimum black-hole radius and mass, and their near-horizon strong dynamics is expected to leave its imprint on a number of observables. The obtained solutions survive only when the synergy of $\phi$ with the GB term is supplemented by the linear Ricci term.

Note added.-Recently, two more works appeared [19,20] that studied the emergence of black-hole solutions with scalar hair in the context of the same theory.

[1] J. D. Bekenstein, Phys. Rev. Lett. 28, 452 (1972); C. Teitelboim, Lett. Nuovo Cimento 3, 397 (1972).

[2] M. S. Volkov and D. V. Galtsov, JETP Lett. 50, 346 (1989); P. Bizon, Phys. Rev. Lett. 64, 2844 (1990); B. R. Greene, S. D. Mathur, and C. M. O’Neill, Phys. Rev. D 47, 2242 (1993); K. I. Maeda, T. Tachizawa, T. Torii, and T. Maki, Phys. Rev. Lett. 72, 450 (1994).

[3] H. Luckock and I. Moss, Phys. Lett. B 176, 341 (1986); S. Droz, M. Heusler, and N. Straumann, Phys. Lett. B 268, 371 (1991).

[4] J. D. Bekenstein, Ann. Phys. (N.Y.) 82, 535 (1974); 91, 75 (1975).

[5] J. D. Bekenstein, Phys. Rev. D 51, R6608 (1995).

[6] C. A. R. Herdeiro and E. Radu, Int. J. Mod. Phys. D 24, 1542014 (2015).

[7] T. P. Sotiriou and V. Faraoni, Phys. Rev. Lett. 108 (2012) 081103.

[8] L. Hui and A. Nicolis, Phys. Rev. Lett. 110, 241104 (2013).

[9] P. Kanti, N. E. Mavromatos, J. Rizos, K. Tamvakis, and E. Winstanley, Phys. Rev. D 54, 5049 (1996); 57, 6255 (1998).

[10] E. Babichev and C. Charmousis, J. High Energy Phys. 08 (2014) 106.

[11] T. P. Sotiriou and S. Y. Zhou, Phys. Rev. Lett. 112, 251102 (2014).

[12] T. P. Sotiriou and S. Y. Zhou, Phys. Rev. D 90, 124063 (2014); R. Benkel, T. P. Sotiriou, and H. Witek, Classical Quantum Gravity 34, 064001 (2017); Phys. Rev. D 94, 121503 (2016).

[13] R. R. Metsaev and A. A. Tseytlin, Nucl. Phys. B293, 385 (1987).

[14] G. W. Horndeski, Int. J. Theor. Phys. 10, 363 (1974).

[15] Note that, in the expression of $e^{B}$, we have kept only the (+) sign as the (-) sign leads to $e^{B} \simeq \mathcal{O}(1)$, which is not a blackhole solution.

[16] J. L. Blazquez-Salcedo et al., IAU Symp. 12, 265 (2016). 
[17] S. Bhattacharya and S. Chakraborty, Phys. Rev. D 95, 044037 (2017); I. Banerjee, S. Chakraborty, and S. SenGupta, Phys. Rev. D 96, 084035 (2017).

[18] J. L. Blazquez-Salcedo, C. F. B. Macedo, V. Cardoso, V. Ferrari, L. Gualtieri, F. S. Khoo, J. Kunz, and P. Pani, Phys. Rev. D 94, 104024 (2016).
[19] D. D. Doneva and S. S. Yazadjiev, following Letter, Phys. Rev. Lett. 120, 131103 (2018).

[20] H. O. Silva, J. Sakstein, L. Gualtieri, T. P. Sotiriou, and E. Berti, this issue, Phys. Rev. Lett. 120, 131104 (2018). 